\title{
BOUNDARY INTEGRAL SOLUTION OF POTENTIAL PROBLEMS ARISING IN THE MODELLING OF ELECTRIFIED OIL FILMS
}

\author{
DAVID J. CHAPPELL
}

Communicated by Francisco-Javier Sayas

\begin{abstract}
We consider a class of potential problems on a periodic half-space for the modeling of electrified oil films, which are used in the development of novel switchable liquid optical devices (diffraction gratings). A boundary integral formulation which reduces the problem to the study of the oil-air interface alone is derived and solved in a highly efficient manner using the Nyström method. The oil films encountered experimentally are typically very thin and thus an interface-only integral representation is important for avoiding the near-singularity problems associated with boundary integral methods for long slender domains. The super-algebraic convergence of the proposed method is discussed and demonstrated via appropriate numerical experiments.
\end{abstract}

1. Introduction. We consider a transmission problem for the Laplace equation in a periodic half-plane for modelling the electric potential on an interface between a thin layer of oil and the surrounding air. A fast solution algorithm is sought for the case where the timedependent interface position is given as the (discrete) data generated from the solution of an associated coupled thin fluid and interface dynamics problem. In particular, the interface motion is a result of dielectrophoresis forces, where the potential is applied via electrodes placed at the base of the oil layer. Dielectrophoresis is defined as the motion of matter caused by polarization effects in a nonuniform electric field $[\mathbf{1 6}, \mathbf{1 7}]$. The use of dielectrophoresis forces to stimulate fluid motion

2010 AMS Mathematics subject classification. Primary 35J05, 45B05, 65N38, $65 \mathrm{R} 20$.

Keywords and phrases. Boundary integral method, transmission problems, Laplace equation, Nyström method, dielectrophoresis.

Received by the editors on April 17, 2014, and in revised form on January 28, 2015. 
has been considered in [11] and is of interest in optics due to potential applications in the development of novel switchable liquid optical devices (diffraction gratings) [5]. In this work, we focus on the solution of the transmission problem for the Laplace equation and solve for the electric potential on the oil-air interface. We also compute the normal and tangential derivatives of the potential on the interface since these are the quantities of interest for the boundary data of the associated thin film fluid problem.

The work here aims to provide a first step in moving from the study of the static problem described in [4] to the dynamic situation described above, via an efficient solution method for the underlying potential problem. Related work on the simulation of electrified fluids has been carried out using a range of techniques including asymptotic approaches $[4,23]$, level set methods [22], finite element approaches for coupled fluid flow and dynamic interface models [9], and boundary integral methods for coupled potential and dynamic interface problems [21].

Our strategy will be to reformulate our transmission problem in a periodic half-plane as a boundary integral problem defined only on the interface. A commonly cited advantage of boundary integral methods is the reduction in dimensionality (by one) to the boundary of the domain being studied. Here, by making explicit use of the half-space solution of a related boundary value problem, we go one step further and reduce our study to the interface part of the boundary. Considering that our goal is the computation of the potential and its normal and tangential derivatives on the interface, this reduction of the problem provides the ideal platform for a highly efficient method. A major advantage over a conventional boundary integral formulation where the entire boundary of the finite domain is discretized, is that the near-singularity problems due to the long slender geometry are completely avoided here.

Two further major reasons for adopting a boundary integral approach are that the relevant Green's functions are available in a simple closed form making it relatively simple to implement a boundary integral method (compared to, say, [21], where the periodic Green's function must be approximated via fast summation methods). Secondly, the infinite domains are dealt with intrinsically in the boundary integral formulation, both along and perpendicular to the direction of periodicity. This means that the imposition of artificial (non-reflecting) boundaries, as would be required for finite element and finite difference 
approaches, is not necessary.

A related class of potential problems can be found in the study of water waves. Preston, Chamberlain and Chandler-Wilde have derived and analyzed a boundary integral formulation for the associated Dirichlet problem on a half-space [18] and presented an efficient and super-algebraically convergent Nyström method for its numerical solution in [19]. These two papers are a subset of a wider body of work on boundary integral methods for scattering from rough surfaces (usually concerned with the solution of the Helmholtz equation), and references can be found within. Boundary integral methods for transmission problems can be found in $[\mathbf{7}, \mathbf{8}, \mathbf{1 0}, \mathbf{1 2}, \mathbf{2 0}]$ and references therein. Work in this area is generally concentrated on the solution of the Helmholtz equation in both the interior and (unbounded) exterior of a two or three-dimensional bounded domain, given some regularity properties on the interface between the interior and exterior domains. Boundary integral methods for transmission problems between various configurations of bounded domains are considered in $[\mathbf{2}, \mathbf{3}, \mathbf{6}]$. The transmission problem for the Laplace equation is also considered explicitly in [7].

The paper is structured as follows. In the next section, we describe the governing transmission problem for the Laplace equation and show that it has at most one solution. In Section 3, we reformulate this problem as a second-kind integral equation on the interface, making use of the solution to the periodic half-space boundary value problem, which plays the role of our boundary data. The Dirichlet-to-Neumann operator for the interface is also introduced, leading to the solution of a first-kind integral equation for the interface Neumann data. Section 4 then describes the numerical solution of these two integral equations via the Nyström method with appropriate quadrature rules. In particular, we draw on the theory presented in $[\mathbf{1}, \mathbf{1 3}, \mathbf{1 4}, \mathbf{1 9}]$ to design a scheme that will exhibit super-algebraic convergence properties. In Section 5, we demonstrate this convergence rate via some numerical experiments with a set of parameters that are appropriate for our application of modelling the potential at the interface between a thin film of oil and the surrounding air.

2. The transmission problem for the Laplace equation. Denote $H=\left\{(x, y) \in \mathbb{R}^{2} \mid y>0\right\}$, and let $h: \mathbb{R} \rightarrow \mathbb{R}$ define an interface at $y=h(x)$ dividing $H$ into $\Omega_{1}=\{(x, y) \in H \mid y<h(x)\}$ and 
$\Omega_{2}=\{(x, y) \in H \mid y>h(x)\}$. Here, $\Omega_{1}$ represents a thin film of oil with boundary $\Gamma_{1}=\Gamma_{I} \cup \Gamma_{0}$, where $\Gamma_{I}=\left\{(x, y) \in \mathbb{R}^{2} \mid y=h(x)\right\}$ and $\Gamma_{0}=\left\{(x, y) \in \mathbb{R}^{2} \mid y=0\right\}$. The domain $\Omega_{2}$ represents the surrounding air and has boundary $\Gamma_{2}=\Gamma_{I}$. We will assume that $h$ is positive and $C^{k}$ for some $k \geq 2$. Let $B C^{k}\left(\bar{\Omega}_{\alpha}\right)$ denote the space of bounded functions in $C^{k}\left(\Omega_{\alpha}\right)$ that can be continuously extended into the closure $\bar{\Omega}_{\alpha}$ with $\alpha=1,2$. Consider the following transmission problem for the electric potential $\phi_{\alpha} \in B C^{2}\left(\bar{\Omega}_{\alpha}\right)$ for $\alpha=1,2$ :

$$
\begin{array}{ll}
\triangle \phi_{\alpha}=0 & \text { in } \Omega_{\alpha}, \\
\phi_{1}=\phi_{2} & \text { on } \Gamma_{I}, \\
\epsilon_{1} \frac{\partial \phi_{1}}{\partial \nu}=\epsilon_{2} \frac{\partial \phi_{2}}{\partial \nu} & \text { on } \Gamma_{I} .
\end{array}
$$

In addition, we prescribe the boundary conditions

$$
\begin{array}{lr}
\phi_{1}(x, y)=f(x) & \text { on } \Gamma_{0}, \\
\frac{\partial \phi_{2}}{\partial y}(x, y) \longrightarrow 0 & \text { as } y \rightarrow \infty .
\end{array}
$$

Here, $\epsilon_{\alpha} \in(0, \infty)$ is the dielectric constant in $\Omega_{\alpha}$ and $\nu$ is the unit normal vector pointing out of $\Omega_{1}$. Under the assumption that $h$ and $f$ are $2 L$-periodic, the problem is reduced to the study of a single periodic cell of $H$ with $-L \leq x<L$. Note that this is a reasonable assumption here since $f$ represents the potential applied by a regularly spaced array of electrodes. The problem setup is shown in Figure 1.

Proposition 2.1. Consider the transmission problem (2.1)-(2.5) with $2 L$-periodic boundary condition $f \in C(\mathbb{R})$ and $2 L$-periodic interface $0<h \in C^{k}(\mathbb{R})$ for some integer $k \geq 2$. If a solution $\phi_{\alpha} \in B C^{2}\left(\bar{\Omega}_{\alpha}\right)$, $\alpha=1,2$ of this problem exists, then it is unique.

Proof. A similar argument to that given for ([7, Proposition 4.7]) can be applied. We actually show that the boundary value problem (2.1)-(2.5) with homogeneous boundary condition $f=0$ only has the trivial solution $\phi_{1}=\phi_{2}=0$, from which the proposition is a straightforward consequence.

Due to the periodicity restriction then a bound of the form $0<$ $h<U$ holds for some constant $U$. Denoting the line $y=U$ by $\Gamma_{U}$ and 


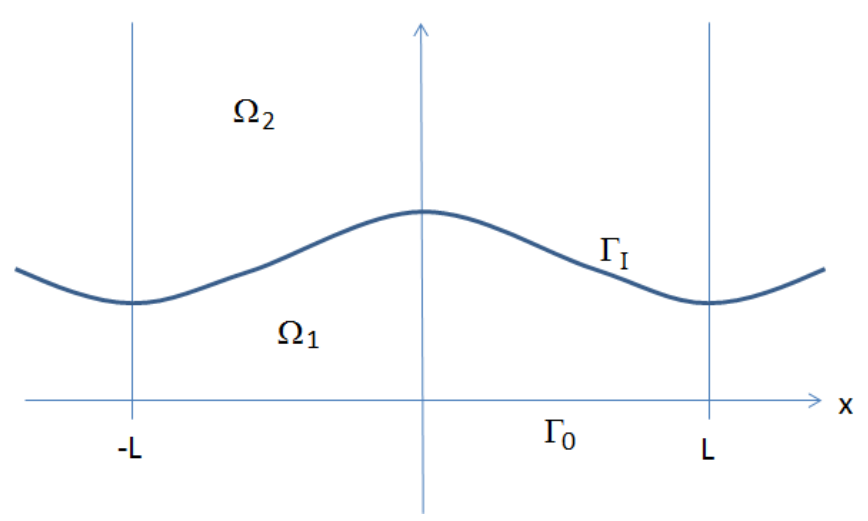

Figure 1. Problem setup.

applying Green's first identity on $\Omega_{1}$ and $\Omega_{2}^{*}=\Omega_{2} \cap\left\{(x, y) \in \mathbb{R}^{2} \mid y<U\right\}$ gives

$$
\begin{aligned}
\int_{\Gamma_{0}} \frac{\partial \phi_{1}}{\partial \nu} \phi_{1} d x+\int_{\Gamma_{I}} \frac{\partial \phi_{1}}{\partial \nu} \phi_{1} d s & =\int_{\Omega_{1}}\left|\nabla \phi_{1}\right|^{2} d A \\
-\int_{\Gamma_{U}} \frac{\partial \phi_{2}}{\partial \nu} \phi_{2} d x-\int_{\Gamma_{I}} \frac{\partial \phi_{2}}{\partial \nu} \phi_{2} d s & =\int_{\Omega_{2}^{*}}\left|\nabla \phi_{2}\right|^{2} d A .
\end{aligned}
$$

Note that periodicity means that the terms from integrating along the vertical sides exactly cancel and the boundary condition $f=0$ means that the integral over $\Gamma_{0}$ vanishes. Combining $(2.6),(2.7)$ and the interface conditions $(2.2),(2.3)$ gives

$$
-\epsilon_{2} \int_{\Gamma_{U}} \frac{\partial \phi_{2}}{\partial \nu} \phi_{2} d x=\epsilon_{2} \int_{\Omega_{2}^{*}}\left|\nabla \phi_{2}\right|^{2} d A+\epsilon_{1} \int_{\Omega_{1}}\left|\nabla \phi_{1}\right|^{2} d A .
$$

Taking the limit as $U \rightarrow \infty$ and using that $\epsilon_{\alpha}>0, \alpha=1,2$, leads to the conclusion that $\phi_{\alpha}, \alpha=1,2$, are both constant functions. The boundary condition (2.4) and the continuity condition (2.2) mean that these constants must both be zero.

In the full multi-physics model, where the potential problem here is coupled with a dynamic fluid-interface model, we wish to study the quasi-time-dependent case where $f$ depends on time in the sense of 
switching the applied potential on or off. This would mean solving for $\phi_{\alpha}$ at a number of time-steps with varying interface position $h$. In this work, we simply consider modelling a static potential for a range of different interface geometries $h$, with the potential switched on. However, we emphasize that the extension of the methods developed here to the dynamic case would be straightforward.

3. Boundary integral formulation. In this section, we recast the transmission problem (2.1)-(2.5) as a boundary integral equation on a single periodic section of the interface $\Gamma_{I}$. From hereon, we make the abuse of notation that $\Gamma_{I}, \Gamma_{0}$ and $\Omega_{\alpha}$ for $\alpha=1,2$; all refer to the restriction of these curves/domains to a single periodic section from $x=-L$ to $x=L$.

3.1. Green's functions, layer potentials and the periodic halfplane solution. A key ingredient in our boundary integral formulation will be the $2 L$-periodic half-plane Green's function for the Laplace equation and its derivative with respect to $\nu$. The periodic Green's function is given as (see, for example, [15])

$$
G\left(\mathbf{x}, \mathbf{x}_{0}\right)=-\frac{1}{2 \pi} \ln \left(2\left|\sin \left(\frac{\pi}{2 L}\left(z-z_{0}\right)\right)\right|\right),
$$

where $\mathbf{x}=(x, y) \in H, z=x+i y, \mathbf{x}_{0}=\left(x_{0}, y_{0}\right) \in \bar{H}$ and $z_{0}=x_{0}+i y_{0}$. It follows from the method of images that the periodic Green's function on $H$ may be written

$$
G_{H}\left(\mathbf{x}, \mathbf{x}_{0}\right)=G\left(\mathbf{x}, \mathbf{x}_{0}\right)-G\left(\mathbf{x}, \mathbf{x}_{0}^{\prime}\right),
$$

where $\mathbf{x}_{0}^{\prime}=\left(x_{0},-y_{0}\right)$ is the mirror image of $\mathbf{x}_{0}$ in $\Gamma_{0}$. Computing the partial derivatives of $G$, we obtain

$$
\begin{aligned}
& \frac{\partial G_{H}}{\partial x_{0}}\left(\mathbf{x}, \mathbf{x}_{0}\right)=\frac{1}{4 L} \Re\left(\cot \left(\frac{\pi}{2 L}\left(z-z_{0}\right)\right)-\cot \left(\frac{\pi}{2 L}\left(z-z_{0}^{\prime}\right)\right)\right), \\
& \frac{\partial G_{H}}{\partial y_{0}}\left(\mathbf{x}, \mathbf{x}_{0}\right)=-\frac{1}{4 L} \Im\left(\cot \left(\frac{\pi}{2 L}\left(z-z_{0}\right)\right)+\cot \left(\frac{\pi}{2 L}\left(z-z_{0}^{\prime}\right)\right)\right),
\end{aligned}
$$


where $z_{0}^{\prime}=x_{0}-i y_{0}$. It is now straightforward from (3.3) and (3.4) to compute the derivative of $G_{H}$ with respect to $\nu_{0}$, the unit normal vector at $\mathbf{x}_{0}$ pointing out of $\Omega_{1}$.

It is convenient here to introduce interface single and double layer potential operators $\mathcal{S}$ and $\mathcal{D}$, respectively, acting on a continuous function $\phi$ at $\mathbf{x} \in \Omega_{\alpha}(\alpha=1,2)$

$$
\begin{aligned}
& \mathcal{S} \phi(\mathbf{x})=\int_{\Gamma_{I}} G_{H}\left(\mathbf{x}, \mathbf{x}_{0}\right) \phi\left(\mathbf{x}_{0}\right) d \Gamma\left(\mathbf{x}_{0}\right) \\
& \mathcal{D} \phi(\mathbf{x})=\int_{\Gamma_{I}} \frac{\partial G_{H}}{\partial \nu_{0}}\left(\mathbf{x}, \mathbf{x}_{0}\right) \phi\left(\mathbf{x}_{0}\right) d \Gamma\left(\mathbf{x}_{0}\right) .
\end{aligned}
$$

In order to distinguish the case when $\mathbf{x} \in \Gamma_{I}$, we rename the interface single and double layer potentials $V$ and $K$, respectively.

Now let us consider the transmission problem (2.1)-(2.5) in the absence of an interface. The problem is simplified to the study of a boundary value problem for the Laplace equation on $\Omega_{1}=H$, with $2 L$-periodic Dirichlet data $f$ along $\Gamma_{0}(2.4)$, and condition (2.5). Using Green's representation formula for $\mathbf{x} \in H$, the solution of this half-plane problem $\phi_{H}$ may be expressed in the form

$$
\begin{aligned}
\phi_{H}(\mathbf{x}) & =\int_{\Gamma_{0}}\left(G_{H}\left(\mathbf{x}, \mathbf{x}_{0}\right) \frac{\partial \phi_{H}}{\partial \nu_{0}}\left(\mathbf{x}_{0}\right)-\frac{\partial G_{H}}{\partial \nu_{0}}\left(\mathbf{x}, \mathbf{x}_{0}\right) f\left(x_{0}\right)\right) d x_{0} \\
& =\int_{\Gamma_{0}} \frac{\partial G_{H}}{\partial y_{0}}\left(\mathbf{x}, \mathbf{x}_{0}\right) f\left(x_{0}\right) d x_{0}
\end{aligned}
$$

The uniqueness of $\phi_{H}$ follows from Proposition 2.1 with $\epsilon_{1}=\epsilon_{2}$. We will make use of the solution $\phi_{H}$ in the next section when deriving the boundary integral formulation for the transmission problem (2.1)-(2.5).

\subsection{Boundary integral formulation for the transmission prob-}

lem. We derive a direct boundary integral formulation for the transmission problem (2.1)-(2.5) using Green's representation formula. This has the advantage, in comparison with indirect formulations, that the unknowns appearing in the boundary integral equations are the physical quantities (potentials) that we wish to compute. We note also that a single layer potential solution ansatz would fail here due to $G_{H}$ vanishing on $\Gamma_{0}$ where the boundary condition $f$ is prescribed. A double layer potential solution ansatz would lead to a more complicated equation for computing the Neumann data on the interface than the method 
proposed here. A further advantage of our direct approach is that it allows our equations to be formulated only on the interface boundary $\Gamma_{I}$, by making use of the half-space solution $\phi_{H}$, as described below. This advantage not only provides efficiency savings through reducing the size of the domain to be discretized, but also means that problems due to near-singularities associated with boundary integral methods in long slender domains are avoided too.

We first apply Green's representation formula in $\Omega_{2}$ to give:

$$
\phi_{2}=\mathcal{D} \phi_{2}-\mathcal{S}\left(\frac{\partial \phi_{2}}{\partial \nu_{0}}\right) .
$$

Note that the integrals as $y \rightarrow \infty$ vanish due to both $\phi_{2}$ and $G_{H}$ remaining bounded as $y \rightarrow \infty$ and satisfying the radiation condition (2.5). Taking the limit as the solution point tends to $\Gamma_{I}$ and applying the interface conditions (2.2) and (2.3) yields

$$
V\left(\frac{\partial \phi_{1}}{\partial \nu_{0}}\right)=\frac{\epsilon_{2}}{\epsilon_{1}}\left(-\frac{I}{2}+K\right) \phi_{1}
$$

The next step is to apply Green's representation formula in $\Omega_{1}$ giving rise to

$$
\phi_{1}(\mathbf{x})=\int_{\Gamma_{1}}\left(G_{H}\left(\mathbf{x}, \mathbf{x}_{0}\right) \frac{\partial \phi_{1}}{\partial \nu_{0}}\left(\mathbf{x}_{0}\right)-\frac{\partial G_{H}}{\partial \nu_{0}}\left(\mathbf{x}, \mathbf{x}_{0}\right) \phi_{1}\left(\mathbf{x}_{0}\right)\right) d \Gamma_{1}\left(\mathbf{x}_{0}\right) .
$$

Splitting the integrals into the sum of an integral over $\Gamma_{I}$ and an integral over $\Gamma_{0}$ yields

$$
\begin{aligned}
\phi_{1}(\mathbf{x})= & \mathcal{S}\left(\frac{\partial \phi_{1}}{\partial \nu_{0}}\right)-\mathcal{D} \phi_{1} \\
& +\int_{\Gamma_{0}}\left(G_{H}\left(\mathbf{x}, \mathbf{x}_{0}\right) \frac{\partial \phi_{1}}{\partial \nu_{0}}\left(\mathbf{x}_{0}\right)-\frac{\partial G_{H}}{\partial \nu_{0}}\left(\mathbf{x}, \mathbf{x}_{0}\right) \phi_{1}\left(\mathbf{x}_{0}\right)\right) d x_{0} .
\end{aligned}
$$

Applying (2.4) and (3.7), then (3.12) simplifies to

$$
\phi_{1}=\mathcal{S}\left(\frac{\partial \phi_{1}}{\partial \nu_{0}}\right)-\mathcal{D} \phi_{1}+\phi_{H}
$$


Taking the limit as the solution point tends to $\Gamma_{I}$ as before and rearranging yields

$$
V\left(\frac{\partial \phi_{1}}{\partial \nu_{0}}\right)=\left(\frac{I}{2}+K\right) \phi_{1}-\phi_{H}
$$

Combining equations (3.10) and (3.14) results in the following secondkind Fredholm integral equation for $\phi=\phi_{1}=\phi_{2}$ on $\Gamma_{I}$

$$
\left(I-2 \frac{\left(\epsilon_{2}-\epsilon_{1}\right)}{\left(\epsilon_{1}+\epsilon_{2}\right)} K\right) \phi=\frac{2 \epsilon_{1}}{\left(\epsilon_{1}+\epsilon_{2}\right)} \phi_{H} .
$$

Clearly, the case $\epsilon_{1}=\epsilon_{2}$ reduces to $\phi=\phi_{H}$ as expected. Note that here we are effectively treating $\phi_{H}$ as our boundary data on $\Gamma_{I}$ and that, since $\phi_{H}$ is harmonic, it is analytic. In the examples considered later, a simple closed form expression will be available for $\phi_{H}$. However, in general, it may be necessary to approximate $\phi_{H}$ by, for example, a truncated Fourier series (if solving the half-plane problem via separation of variables) or quadrature (if computing $\phi_{H}$ directly from the boundary integral formula (3.8)).

Let us now consider the operator $K$ in further detail. Since, for $\mathbf{x} \in \Gamma_{I}$, then $(x, y)=(x, h(x))$, then if we also have $\mathbf{x}_{0} \in \Gamma_{I}$, we may write

$$
T_{H}\left(x, x_{0}\right):=\frac{\partial G_{H}}{\partial \nu_{0}}\left(\mathbf{x}, \mathbf{x}_{0}\right)
$$

and hence, with a slight abuse of notation,

$$
K \phi(x)=\int_{-L}^{L} T_{H}\left(x, x_{0}\right) \phi\left(x_{0}\right) \sqrt{1+h^{\prime}\left(x_{0}\right)^{2}} d x_{0} .
$$

Considering, therefore, a single periodic strip with $x \in[-L, L]$, then the behavior of $K$ close to $x=x_{0}$ is identical to that for the double layer potential on a simple closed curve studied in ([1, Chapter 7$])$. This is evident from taking limits in (3.3) and (3.4) as $\left(x-x_{0}\right) \rightarrow 0$. Since $h \in C^{k}([-L, L])$ for some $k \geq 2$, then it follows from ([1, Chapter 7]) that

$$
T_{H} \in C^{k-2}([-L, L] \times[-L, L]) .
$$

As a result of this, $K$ is a compact operator on $C([-L, L])$ with the maximum norm (see for example, [13, Theorem 2.21]). Using the theory of compact integral operators [13], we now prove the existence 
and uniqueness of solutions to the integral equation (3.15). We first prove the following:

$$
\begin{aligned}
f=0 & \Longleftrightarrow \phi_{H}=0 \\
& \Longleftrightarrow \phi=0 .
\end{aligned}
$$

The implications $f=0 \Rightarrow \phi_{H}=0$ and $f=0 \Rightarrow \phi=0$ are due to the uniqueness of solution to both the transmission problem (Proposition 2.1) and the periodic half-plane problem, which follows from Proposition 2.1 with $\epsilon_{1}=\epsilon_{2}$. The implication $\phi_{H}=0 \Leftarrow \phi=0$ follows from (3.15). Finally, $f=0 \Leftarrow \phi_{H}=0$ relies on the fact that $\phi_{H}$ can be continuously extended to $\Gamma_{0}([\mathbf{1 8}$, Theorem 2.3]) so that

$$
\lim _{y \rightarrow 0} \phi_{H}(\mathbf{x})=f(x),
$$

which may be derived from the jump properties of the double layer potential (3.8).

Hence, assuming $\phi_{H}=0$, then (3.19) gives that $\phi=0$ is the only solution of

$$
\left(I-2 \frac{\left(\epsilon_{2}-\epsilon_{1}\right)}{\left(\epsilon_{1}+\epsilon_{2}\right)} K\right) \phi=0 .
$$

Hence, the operator

$$
I-2 \frac{\left(\epsilon_{2}-\epsilon_{1}\right)}{\left(\epsilon_{1}+\epsilon_{2}\right)} K
$$

is injective on $C([-L, L])$. It now follows from $([\mathbf{1 3}$, Theorem 3.4]) that (3.15) is uniquely solvable and that the solution depends continuously on $\phi_{H}$.

Theorem 3.1. The integral operator $I-2 \mu K: C([-L, L]) \rightarrow$ $C([-L, L])$ is invertible with bounded inverse for any $|\mu|<1$. Consequently, there exists a unique solution $\phi_{\alpha} \in B C^{2}\left(\bar{\Omega}_{\alpha}\right)$ to the transmission problem (2.1)-(2.5) considered in Proposition 1.

3.3. The Dirichlet-to-Neumann (DtN) operator. For our application in tracking the dynamic evolution of a thin film of oil, the boundary data for the fluid equations will depend on the normal and tangential derivatives of $\phi$ on $\Gamma_{I}$. Furthermore, the normal derivative would be needed for computing the domain potentials via (3.9) and (3.13). 
Later, we discuss interpolation formulae for obtaining the tangential derivative. The normal derivative will be computed using the DtN operator. Combining (2.3) and (3.10) yields the following first-kind integral equation for $\partial \phi_{\alpha} / \partial \nu_{0}, \alpha=1,2$ :

$$
V\left(\frac{\partial \phi_{\alpha}}{\partial \nu_{0}}\right)=\frac{\epsilon_{2}}{\epsilon_{\alpha}}\left(-\frac{I}{2}+K\right) \phi
$$

The DtN operator will be bounded as a map from $H^{1 / 2}\left(\Gamma_{I}\right)$ to $H^{-1 / 2}\left(\Gamma_{I}\right)$ if we can prove the invertibility of $V: H^{-1 / 2}\left(\Gamma_{I}\right) \rightarrow$ $H^{1 / 2}\left(\Gamma_{I}\right)$ with bounded inverse, where $H^{ \pm 1 / 2}$ are the usual Sobolev spaces $([\mathbf{1 3}$, subsection 8.2$])$. To prove this, we need to show that $V$ is bounded and elliptic so the Lax-Milgram theorem gives that $V^{-1}: H^{1 / 2}\left(\Gamma_{I}\right) \rightarrow H^{-1 / 2}\left(\Gamma_{I}\right)$ is bounded. The proof that $V$ : $H^{-1 / 2}\left(\Gamma_{I}\right) \rightarrow H^{1 / 2}\left(\Gamma_{I}\right)$ is bounded can be done using a similar argument to the one presented in $([\mathbf{1 3}$, Theorem 8.23]) for the single layer potential on a simple closed $C^{k}$ curve in $\mathbb{R}^{2}$. The proof of ellipticity is again similar to that for the case of a closed curve in $\mathbb{R}^{2}$. A rough outline restricted to $V: H_{*}^{-1 / 2}\left(\Gamma_{I}\right) \rightarrow H^{1 / 2}\left(\Gamma_{I}\right)$, where

$$
H_{*}^{-1 / 2}\left(\Gamma_{I}\right)=\left\{\widetilde{\sigma} \in H^{-1 / 2}\left(\Gamma_{I}\right) \mid \int_{\Gamma_{I}} \tilde{\sigma} d s=0\right\},
$$

is presented below.

Let $\phi=\mathcal{S} \sigma$, where $\phi_{\alpha}=\left.\phi\right|_{\Omega_{\alpha}}$ for $\alpha=1,2$ and $\sigma$ is a so-called layer density to be determined. The definition of $\mathcal{S}$ allows us to deduce that $\left.\phi\right|_{\Gamma_{0}}=0$ and

$$
\lim _{y \rightarrow \infty} \frac{\partial \phi_{2}}{\partial y}=0
$$

Furthermore, $V$ and its normal derivative will satisfy the standard jump relations associated with the classical single layer potential operator for the Laplace equation in two dimensions. This is simply a consequence of the fact that $G_{H}$ is constructed using the method of images to both incorporate periodicity and to restrict to a half-plane. The resulting additional contributions to the classical operator and their normal derivatives will all be continuous across $\Gamma_{I}$ (on the periodic section considered). Hence, on $\Gamma_{I}$, we have that $\phi_{1}=\phi_{2}$ and

$$
\frac{\partial \phi_{1}}{\partial \nu}-\frac{\partial \phi_{2}}{\partial \nu}=\sigma
$$


From the above, it is then straightforward to deduce that

$$
\int_{\Gamma_{I}}\left(\phi_{1} \frac{\partial \phi_{1}}{\partial \nu}-\phi_{2} \frac{\partial \phi_{2}}{\partial \nu}\right) d s=\int_{\Gamma_{I}} \sigma V \sigma d s .
$$

Arguing as in the proof of Proposition 2.1 using Green's first identity, (3.23) and that $\left.\phi\right|_{\Gamma_{0}}=0$, leads to

$$
\int_{\Gamma_{I}} \phi_{\alpha} \frac{\partial \phi_{\alpha}}{\partial \nu} d s=(-1)^{\alpha-1} \int_{\Omega_{\alpha}}\left|\nabla \phi_{\alpha}\right|^{2} d A .
$$

Combining (3.25) and (3.26), we find

$$
\begin{aligned}
\int_{\Gamma_{I}} \sigma V \sigma d s & =\int_{\Omega_{1}}\left|\nabla \phi_{1}\right|^{2} d A+\int_{\Omega_{2}}\left|\nabla \phi_{2}\right|^{2} d A \\
& =\left|\phi_{1}\right|_{H^{1}\left(\Omega_{1}\right)}^{2}+\left|\phi_{2}\right|_{H^{1}\left(\Omega_{2}\right)}^{2} .
\end{aligned}
$$

Now, considering the norm of the layer density, we have

$$
\begin{aligned}
\|\sigma\|_{H^{-1 / 2}\left(\Gamma_{I}\right)}^{2} & \left.=\left\|\frac{\partial \phi_{1}}{\partial \nu}-\frac{\partial \phi_{2}}{\partial \nu}\right\|_{H^{-1 / 2}\left(\Gamma_{I}\right)}^{2}+\left\|\frac{\partial \phi_{2}}{\partial \nu}\right\|_{H^{-1 / 2}\left(\Gamma_{I}\right)}^{2}\right) \\
& \leq 2\left(\left\|\frac{\partial \phi_{1}}{\partial \nu}\right\|_{H^{-1 / 2}\left(\Gamma_{I}\right)}^{2} \|\right. \\
& \leq C\left(\left|\phi_{1}\right|_{H^{1}\left(\Omega_{1}\right)}^{2}+\left|\phi_{2}\right|_{H^{1}\left(\Omega_{2}\right)}^{2}\right) .
\end{aligned}
$$

The final inequality follows by applying the Cauchy-Schwarz inequality in the first Green identity to get

$$
\left|\int_{\Gamma_{I}} \psi \frac{\partial \phi_{\alpha}}{\partial \nu} d s\right| \leq\left|\phi_{\alpha}\right|_{H^{1}\left(\Omega_{\alpha}\right)}|\psi|_{H^{1}\left(\Omega_{\alpha}\right)},
$$

with $\psi=\mathcal{S} \widetilde{\sigma}$ for some $\widetilde{\sigma} \in H_{*}^{-1 / 2}\left(\Gamma_{I}\right)$. Then, applying the inverse trace theorem to $\left.\psi\right|_{\Gamma_{I}} \in H^{1 / 2}\left(\Gamma_{I}\right)$ and using duality of $H^{ \pm 1 / 2}\left(\Gamma_{I}\right)$, leads to an inequality of the form

$$
\begin{aligned}
&\left\|\frac{\partial \phi_{\alpha}}{\partial \nu}\right\|_{H^{-1 / 2}\left(\Gamma_{I}\right)}=\sup _{\psi \in H^{1 / 2}\left(\Gamma_{I}\right) \backslash\{0\}} \frac{\left|\int_{\Gamma_{I}} \psi \frac{\partial \phi_{\alpha}}{\partial \nu} d s\right|}{\|\psi\|_{H^{1 / 2}\left(\Gamma_{I}\right)}} \\
& \leq C_{\alpha}\left|\phi_{\alpha}\right|_{H^{1}\left(\Omega_{\alpha}\right)},
\end{aligned}
$$

where $C$ and $C_{\alpha}$ are positive constants with $C=2 \max \left(C_{\alpha}\right)^{2}$. To conclude the argument, we combine (3.27) and (3.28) to give ellipticity, 
that is,

$$
C \int_{\Gamma_{I}} \sigma V \sigma d s \geq\|\sigma\|_{H^{-1 / 2}\left(\Gamma_{I}\right)}^{2}
$$

4. Discretization using the Nyström method. In this section, we describe the discretization of the integral equations (3.15) and (3.22) using the Nyström method with suitable quadratures. In particular, we draw on the theory presented in $[\mathbf{1}, \mathbf{1 3}, \mathbf{1 4}, \mathbf{1 9}]$ in order to design a method with super-algebraic convergence, as demonstrated by the numerical results in the next section. It should be noted that, to obtain these convergence rates, requires that $h \in C^{\infty}([-L, L])$, and so from hereon we make this assumption. In the application to modelling the potential in a layer of oil, where the (time-dependent) oil-air interface position will only be given as a set of equi-spaced coordinates, we will use interpolation by trigonometric polynomials to ensure the required smoothness. This is a good choice of the interpolation scheme due to the periodicity of $h$, and the availability of a highly efficient FFT based implementation. Even though $h$ will be given explicitly in the examples here, we will interpolate anyway to ensure the algorithm can be applied more generally.

4.1. Discretization for a smooth kernel. Let us first consider the second-kind equation (3.15). Under the assumption of an infinitely differentiable interface, we also have an infinitely differentiable kernel and, as discussed before, the data term $\phi_{H}$ is also infinitely differentiable. In this situation, a simple application of the trapezoidal rule yields a super-algebraically convergent method $[\mathbf{1}, \mathbf{1 9}]$. To implement this scheme, we first use that $\mathbf{x}=(x, h(x))$ on $\Gamma_{I}$ and write integrals over $\Gamma_{I}$ in the form

$$
\int_{\Gamma_{I}} F(\mathbf{x}) d \Gamma(\mathbf{x})=\int_{-L}^{L} \widetilde{F}(x) \sqrt{1+h^{\prime}(x)^{2}} d x .
$$

Applying the trapezoidal rule with $n$ subintervals gives

$$
\int_{\Gamma_{I}} F(\mathbf{x}) d \Gamma(\mathbf{x}) \approx \frac{2 L}{n} \sum_{j=1}^{n} \widetilde{F}\left(x_{j}\right) \sqrt{1+h^{\prime}\left(x_{j}\right)^{2}},
$$

where $x_{j}=-L+2 L(j-1) / n$. Note that, because we have assumed above that $h$ is given by trigonometric interpolation, its derivative 
may be computed simply by differentiating its Fourier components as described in [19].

Applying the formula (4.2) to the integral in the definition of $K$ yields the following approximation

$$
K \phi\left(x_{i}, h\left(x_{i}\right)\right) \approx \frac{2 L}{n} \sum_{j=1}^{n} k_{i, j} \phi\left(x_{j}\right) \sqrt{1+h^{\prime}\left(x_{j}\right)^{2}},
$$

where

$$
k_{i, j}=T_{H}\left(x_{i}, x_{j}\right) .
$$

The following explicit formula for $k_{j, j}$ may be derived (for similar derivations, see [1, Chapter 7] and [19]):

$$
k_{j, j}=\frac{h^{\prime \prime}\left(x_{j}\right)}{4 \pi\left(1+h^{\prime}\left(x_{j}\right)^{2}\right)}+\frac{1}{4 L \sqrt{1+h^{\prime}\left(x_{j}\right)^{2}}} \operatorname{coth}\left(\frac{\pi}{L} h\left(x_{j}\right)\right) .
$$

As with the first derivative, $h^{\prime \prime}$ may be computed simply by differentiating the Fourier components for $h$ twice. For $i \neq j$, we can use the formula obtained directly from (3.3) and (3.4), that is,

$$
\begin{aligned}
k_{i, j}= & \frac{1}{4 L \sqrt{1+h^{\prime}\left(x_{j}\right)^{2}}}\left(h ^ { \prime } ( x _ { j } ) \Re \left(\cot \left(\frac{\pi}{2 L}\left(z_{i}-z_{j}^{\prime}\right)\right)\right.\right. \\
& \left.\quad-\cot \left(\frac{\pi}{2 L}\left(z_{i}-z_{j}\right)\right)\right) \\
- & \left.\left(\cot \left(\frac{\pi}{2 L}\left(z_{i}-z_{j}\right)\right)+\cot \left(\frac{\pi}{2 L}\left(z_{i}-z_{j}^{\prime}\right)\right)\right)\right) .
\end{aligned}
$$

Here we have denoted $z_{j}=x_{j}+i h\left(x_{j}\right)$ and $z_{j}^{\prime}=x_{j}-i h\left(x_{j}\right)$. Notice that (4.5) illustrates the smoothness result (3.18). Applying the approximation (4.3) to the second-kind integral equation (3.15) leads to the following approximate Nyström scheme for the approximate solution $\phi^{n}$

$$
\begin{aligned}
\phi^{n}\left(x_{i}\right)+\frac{4 L\left(\epsilon_{1}-\epsilon_{2}\right)}{n\left(\epsilon_{1}+\epsilon_{2}\right)} \sum_{j=1}^{n} k_{i, j} \phi^{n}\left(x_{j}\right) & \sqrt{1+h^{\prime}\left(x_{j}\right)^{2}} \\
& =\frac{2 \epsilon_{1}}{\left(\epsilon_{1}+\epsilon_{2}\right)} \phi_{H}\left(x_{i}, h\left(x_{i}\right)\right)
\end{aligned}
$$


for $i=1, \ldots, n$. The super-algebraic convergence of $\phi^{n}$ to $\phi$ for increasing $n$ is a consequence of ([19, Theorem 3.12]).

\subsection{Discretization for a kernel with a logarithmic singularity.} In this section, we consider a Nyström method for the solution of equation (3.22), which is a first-kind integral equation for $\partial \phi_{\alpha} / \partial \nu_{0}$, $\alpha=1,2$. In particular, we note that the kernel function $G_{H}$ of the operator $V$ contains a logarithmic singularity and may be written in the form

$$
G_{H}\left(\mathbf{x}, \mathbf{x}_{0}\right)=-\frac{1}{4 \pi} \ln \left(4 \sin ^{2}\left(\frac{\pi}{2 L}\left(x-x_{0}\right)\right)\right)+\widetilde{G}\left(x, x_{0}\right)-G\left(\mathbf{x}, \mathbf{x}_{0}^{\prime}\right),
$$

with

$$
\begin{aligned}
\widetilde{G}\left(x, x_{0}\right)= & -\frac{1}{4 \pi}\left\{\ln \left(4\left(\sin ^{2}\left(\frac{\pi}{2 L}\left(x-x_{0}\right)\right)+\sinh ^{2}\left(\frac{\pi}{2 L}\left(h(x)-h\left(x_{0}\right)\right)\right)\right)\right)\right. \\
& \left.-\ln \left(4 \sin ^{2}\left(\frac{\pi}{2 L}\left(x-x_{0}\right)\right)\right)\right\} .
\end{aligned}
$$

On $\Gamma_{I}$ where $y=h(x)$ for $h \in C^{\infty}([-L, L])$, then both $\widetilde{G}\left(x, x_{0}\right)$ and $G\left(\mathbf{x}, \mathbf{x}_{0}^{\prime}\right)$ are infinitely differentiable with respect to $x, x_{0} \in[-L, L]$. In particular, the diagonal term for $\widetilde{G}$ is given by

$$
\widetilde{G}(x, x)=-\frac{1}{4 \pi} \ln \left(1+h^{\prime}(x)^{2}\right) .
$$

Note that (4.7) implies that the first-kind equation (3.22) falls into the class of first kind equations analyzed in [14]. We therefore base our quadrature method here on the approach suggested in [14] (see also [13] and references therein). Super-algebraic convergence rates will then be attained due to $([\mathbf{1 4}$, Theorem 2.3]) if the right hand side of (3.22) is infinitely differentiable. Note, however, that since in general only an approximate numerical solution of (3.15) will be available, then $\phi$ will need to be replaced by $\phi^{n}$ in (3.22). In order to satisfy the necessary criteria for the super-algebraic convergence rates predicted by ([14, Theorem 2.3]), we may interpolate $\phi^{n}$ using trigonometric polynomials to obtain an infinitely differentiable interpolant. The right hand side of (3.22) may then be written $\epsilon_{2}\left(-\phi^{n}+2 K \phi^{n}\right) /\left(2 \epsilon_{\alpha}\right)$, which 
is clearly infinitely differentiable since

$$
\frac{d^{m}}{d x^{m}}\left(K \phi^{n}\right)(\mathbf{x})=\int_{-L}^{L} \frac{\partial^{m}}{\partial x^{m}} T_{H}\left(x, x_{0}\right) \phi^{n}\left(x_{0}\right) \sqrt{1+h^{\prime}\left(x_{0}\right)^{2}} d x_{0},
$$

and $T_{H}$ is infinitely differentiable for $h \in C^{\infty}([-L, L])$.

We now outline the quadrature rule we employ to approximate the interface single layer potential $V$. For the term containing a logarithmic singularity, we employ a quadrature rule of the form

$$
\begin{aligned}
-\frac{1}{4 \pi} \int_{-L}^{L} \ln \left(4 \sin ^{2}\left(\frac{\pi}{2 L}\left(x-x_{0}\right)\right)\right) F\left(x_{0}\right) & d x_{0} \\
& \approx \sum_{j=1}^{2 N} R_{j}(x) F\left(x_{j}\right)
\end{aligned}
$$

for positive integer $N=n / 2$ (assuming $n$ is even), with $x_{j}=-L+$ $L(j-1) / n, j=1, \ldots, n$, as before. The quadrature weight function $R_{j}(x)$ is given by:

$$
\begin{aligned}
& R_{j}(x) \\
= & \frac{L}{2 \pi n}\left\{\sum_{m=1}^{N-1} \frac{1}{m} \cos \left(\frac{m \pi}{L}\left(x-x_{j}\right)\right)+\frac{1}{n} \cos \left(\frac{N \pi}{L}\left(x-x_{j}\right)\right)\right\} .
\end{aligned}
$$

This choice of quadrature computes the integral in (4.10) exactly when $F$ has been replaced by its trigonometric interpolation polynomial. To see this, replace $F$ in (4.10) by the Lagrange trigonometric polynomial of order $j$, then the formula (4.11) may be derived for the integral on the left hand side; see ([13, page 208]) and [14] for details.

The remaining integrals involved in approximating $V$ have smooth kernels and thus may be well-approximated using the trapezoidal rule as before. We, therefore, arrive at the following approximation for the interface single layer potential

$$
V \frac{\partial \phi_{\alpha}}{\partial \nu_{0}}\left(x_{i}, h\left(x_{i}\right)\right) \approx \sum_{j=1}^{n}\left(R_{j}\left(x_{i}\right)+\frac{2 L}{n} s_{i, j}\right) \frac{\partial \phi_{\alpha}}{\partial \nu_{0}}\left(x_{j}\right) \sqrt{1+h^{\prime}\left(x_{j}\right)^{2}}
$$

for $\alpha=1,2$ and $i=1, \ldots, n$. Here $s_{i, j}$ is the smooth part of the 
integrand for $V$ given by

$$
\begin{aligned}
s_{i, j}= & -\frac{1}{4 \pi}\left\{\operatorname { l n } \left(4 \left(\sin ^{2}\left(\frac{\pi}{2 L}\left(x_{i}-x_{j}\right)\right)\right.\right.\right. \\
& \left.\left.+\sinh ^{2}\left(\frac{\pi}{2 L}\left(h\left(x_{i}\right)-h\left(x_{j}\right)\right)\right)\right)\right) \\
& -\ln \left(4 \left(\sin ^{2}\left(\frac{\pi}{2 L}\left(x_{i}-x_{j}\right)\right)\right.\right. \\
& \left.\left.+\sinh ^{2}\left(\frac{\pi}{2 L}\left(h\left(x_{i}\right)+h\left(x_{j}\right)\right)\right)\right)\right) \\
& \left.-\ln \left(4 \sin ^{2}\left(\frac{\pi}{2 L}\left(x_{i}-x_{j}\right)\right)\right)\right\},
\end{aligned}
$$

for $i \neq j$, and

$$
s_{j, j}=-\frac{1}{4 \pi}\left\{\ln \left(1+h^{\prime}\left(x_{j}\right)^{2}\right)-\ln \left(4 \sinh ^{2}\left(\frac{\pi}{L} h\left(x_{j}\right)\right)\right)\right\} .
$$

Applying the approximation (4.12) to the first-kind integral equation (3.22) leads to the following Nyström scheme for the approximate solution $\partial \phi_{\alpha}^{n} / \partial \nu_{0}$

$$
\begin{aligned}
\sum_{j=1}^{n}\left(R_{j}\left(x_{i}\right)+\frac{2 L}{n} s_{i, j}\right) \frac{\partial \phi_{\alpha}^{n}}{\partial \nu_{0}}\left(x_{j}\right) \sqrt{1+h^{\prime}\left(x_{j}\right)^{2}} \\
=\frac{\epsilon_{2}}{\epsilon_{\alpha}}\left(\frac{-\phi^{n}\left(x_{i}\right)}{2}+\frac{2 L}{n} \sum_{j=1}^{n} k_{i, j} \phi^{n}\left(x_{j}\right) \sqrt{1+h^{\prime}\left(x_{j}\right)^{2}}\right),
\end{aligned}
$$

for $i=1, \ldots, n$ and $\alpha=1,2$. Here $\phi^{n}$ and $k_{i, j}$ are computed as described in the previous section.

5. Numerical experiments. We test our boundary integral model and numerical solution scheme using the same boundary condition for (2.4) as employed in [4] for a static applied potential $f(x)=$ $A \cos (\pi x / L)$, where $A$ is a constant amplitude term. Note that, one can then derive the half-space solution $\phi_{H}$, either using separation of variables or directly from the boundary integral formula (3.8), to get

$$
\phi_{H}(\mathbf{x})=A \cos (\pi x / L) \exp (-\pi y / L) .
$$


In addition, we set the parameter $\epsilon_{1}=8$ and $\epsilon_{2}=1$ to reflect the values for oil and air in our application of interest [4]. For the interface position $h(x)$, we consider three possibilities:

I1. A constant profile $h(x)=h_{0}$ since in this case the results can be checked against an exact solution.

I2. A single period sine curve $h(x)=h_{0}(1+0.2 \sin (\pi x / L))$ of mean height $h_{0}$, similar to that used for numerical tests in [19].

I3. A double period cosine curve $h(x)=h_{0}(1-\cos (2 \pi x / L) /(2 \pi))$ as considered in [4, Section II], which is representative of a typical interface geometry that would be encountered in application to an electrified oil-air interface.

Note that, to allow the input of numeric interface position data, trigonometric interpolation is used to represent the interface position (exactly for the examples above), and derivatives of $h$ are computed via differentiation of its Fourier components as described before.

We use the methods described in the previous section to approximate the interface potential $\phi$ by $\phi^{n}$ and the normal derivative $\partial \phi_{\alpha} / \partial \nu$ by $\partial \phi_{\alpha}^{n} / \partial \nu(\alpha=1,2)$. We also approximate the tangential derivative $\partial \phi_{\alpha} / \partial \tau$, where $\tau$ denotes the tangent vector to $\Gamma_{I}$. To do this, we first compute the derivative with respect to $x$ by applying trigonometric interpolation to $\phi^{n}$ using an FFT and then differentiating the Fourier components (exactly the same as the procedure we carry out for the interface position function $h$ ). One then obtains the tangential derivative by correcting for arc-length via division by a factor of $\sqrt{1+h^{\prime}(x)^{2}}$. A similar procedure is described and rigorously analyzed in [19], where it is shown super-algebraic convergence will also be achieved in the approximation of $\partial \phi_{\alpha} / \partial \tau$ by $\partial \phi_{\alpha}^{n} / \partial \tau, \alpha=1,2$. Note that here the continuity of $\partial \phi_{\alpha}^{n} / \partial \tau$ across $\Gamma_{I}$ follows from the continuity of $\phi^{n}$ across $\Gamma_{I}$, so we may simply write $\partial \phi^{n} / \partial \tau$ for both $\partial \phi_{\alpha}^{n} / \partial \tau$, $\alpha=1,2$.

In the case of interface I1, an analytic solution to the transmission problem (2.1)-(2.5) with boundary data $f(x)=A \cos (\pi x / L)$ can be derived using separation of variables to give

$$
\phi_{1}(\mathbf{x})=\frac{A \cos (\pi x / L)\left(\epsilon_{1}-\epsilon_{2}\right) \exp (\pi y / L)}{\left(\epsilon_{1}+\epsilon_{2}\right) \exp \left(2 \pi h_{0} / L\right)+\left(\epsilon_{1}-\epsilon_{2}\right)}
$$




$$
\begin{aligned}
+ & \frac{A \cos (\pi x / L)\left(\epsilon_{1}+\epsilon_{2}\right) \exp \left(\left(2 h_{0}-y\right) \pi / L\right)}{\left(\epsilon_{1}+\epsilon_{2}\right) \exp \left(2 \pi h_{0} / L\right)+\left(\epsilon_{1}-\epsilon_{2}\right)}, \\
\phi_{2}(\mathbf{x})= & \frac{2 A \epsilon_{1} \cos (\pi x / L) \exp \left(\left(2 h_{0}-y\right) \pi / L\right)}{\left(\epsilon_{1}+\epsilon_{2}\right) \exp \left(2 \pi h_{0} / L\right)+\left(\epsilon_{1}-\epsilon_{2}\right)} .
\end{aligned}
$$

From the solution above, it is easy to calculate $\phi$ and its tangential and normal derivatives, which here correspond to partial derivatives with respect to $x$ and $y$, for interface I1. A plot of $\phi$ for $A=L=1$ and a thin film with $h_{0}=0.03 L$ (comparable to the parameter choice of [4]) is given in Figure 2. The tangential and normal derivatives are also shown.

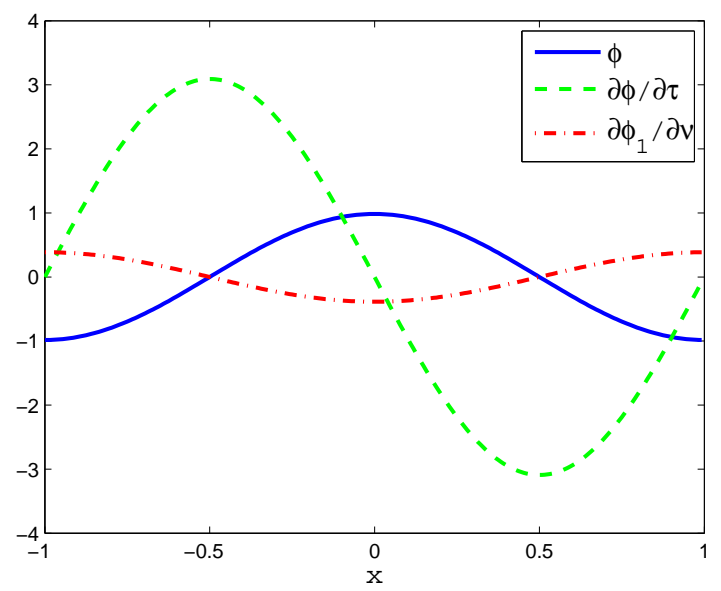

FIGURE 2. Analytic solution of the potential and its tangential and normal derivatives on a flat interface.

We use these analytic solutions to compute the discrete $l_{2}$ error in our numerical approximations of $\phi, \partial \phi / \partial \tau$ and $\partial \phi / \partial \nu$ with the above choice of parameters. The results are shown in Figure 3 and demonstrate the predicted super-algebraic convergence since the rate of convergence speeds up as $n$ is increased, until rounding errors become significant for $n>128$. The results for $\phi$ and $\partial \phi / \partial \tau$ are almost identical until the rounding errors dominate, and then the errors for $\partial \phi / \partial \tau$ and $\partial \phi / \partial \nu$ are very similar. The general trends are very similar to those shown in the Dirichlet problem studied in [19]. 


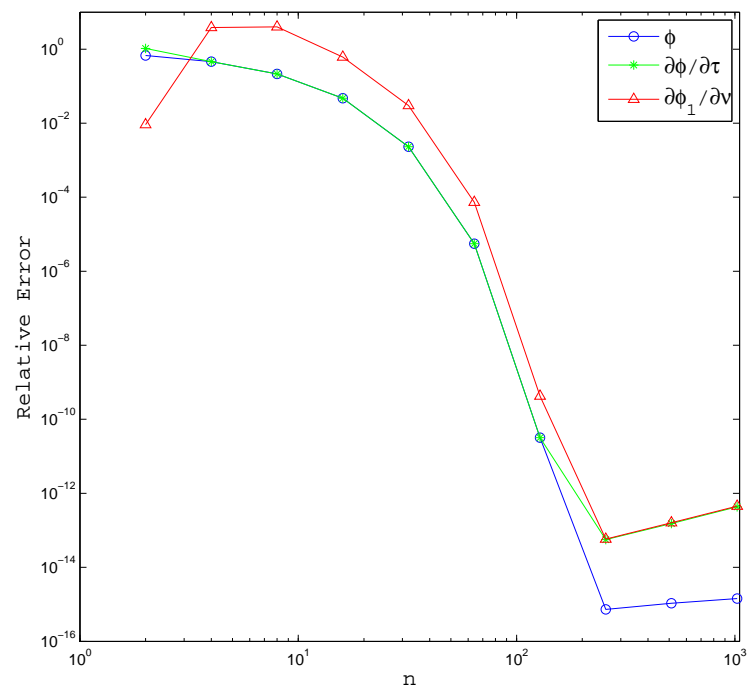

FIgURE 3. Relative errors in the potential and its tangential and normal derivatives on a flat interface.

TABLE 1. Relative errors and estimated orders of convergence for interface I1.

\begin{tabular}{ccccccc}
\hline \multicolumn{2}{c}{ Results for $\phi^{n}$} & \multicolumn{2}{c}{ Results for $\partial \phi^{n} / \partial \tau$} & \multicolumn{2}{c}{ Results for $\partial \phi_{1}^{n} / \partial \nu$} \\
\hline$n$ & Error & EOC & Error & EOC & Error & EOC \\
\hline 16 & $4.506 \mathrm{e}-02$ & - & $4.506 \mathrm{e}-02$ & - & 0.6025 & - \\
32 & $2.307 \mathrm{e}-03$ & 4.29 & $2.307 \mathrm{e}-03$ & 4.29 & $2.983 \mathrm{e}-02$ & 4.34 \\
64 & $5.551 \mathrm{e}-06$ & 8.70 & $5.551 \mathrm{e}-06$ & 8.70 & $7.279 \mathrm{e}-05$ & 8.68 \\
128 & $3.199 \mathrm{e}-11$ & 17.40 & $3.199 \mathrm{e}-11$ & 17.40 & $4.227 \mathrm{e}-10$ & 17.39 \\
256 & $1.187 \mathrm{e}-15$ & 14.72 & $1.611 \mathrm{e}-13$ & 7.63 & $1.566 \mathrm{e}-13$ & 11.40 \\
512 & $1.769 \mathrm{e}-15$ & -0.58 & $4.579 \mathrm{e}-13$ & -1.51 & $4.724 \mathrm{e}-13$ & -1.59 \\
\hline
\end{tabular}

Since analytic solutions are not available for the cases of interface I2 and I3, we compute the discrete $l_{2}$ relative errors and estimate their rates of convergence by using $\phi^{2 n}, \partial \phi^{2 n} / \partial \tau$ and $\partial \phi^{2 n} / \partial \nu$ in the role of the 'exact' solution for $\phi^{n}, \partial \phi^{n} / \partial \tau$ and $\partial \phi^{n} / \partial \nu$, respectively (using only the coincident data points). For consistency, we also 
TABLE 2. Relative errors and estimated orders of convergence for interface I2.

\begin{tabular}{ccccccc}
\hline & \multicolumn{2}{c}{ Results for $\phi^{n}$} & \multicolumn{2}{c}{ Results for $\partial \phi^{n} / \partial \tau$} & \multicolumn{2}{c}{ Results for $\partial \phi_{1}^{n} / \partial \nu$} \\
\hline$n$ & Error & EOC & Error & EOC & Error & EOC \\
\hline 16 & $4.872 \mathrm{e}-02$ & - & $5.462 \mathrm{e}-02$ & - & $7.231 \mathrm{e}-01$ & - \\
32 & $3.248 \mathrm{e}-03$ & 3.91 & $4.610 \mathrm{e}-03$ & 3.57 & $4.832 \mathrm{e}-02$ & 3.90 \\
64 & $1.721 \mathrm{e}-05$ & 7.56 & $3.410 \mathrm{e}-05$ & 7.08 & $2.806 \mathrm{e}-04$ & 7.43 \\
128 & $7.230 \mathrm{e}-10$ & 14.54 & $2.055 \mathrm{e}-09$ & 14.02 & $1.293 \mathrm{e}-08$ & 14.41 \\
256 & $1.861 \mathrm{e}-15$ & 18.57 & $2.692 \mathrm{e}-13$ & 12.90 & $3.476 \mathrm{e}-13$ & 15.18 \\
512 & $2.786 \mathrm{e}-15$ & -0.58 & $7.637 \mathrm{e}-13$ & -1.50 & $1.046 \mathrm{e}-12$ & -1.59 \\
\hline
\end{tabular}

TABLE 3. Relative errors and estimated orders of convergence for interface I3.

\begin{tabular}{ccccccc}
\hline & \multicolumn{2}{c}{ Results for $\phi^{n}$} & \multicolumn{2}{c}{ Results for $\partial \phi^{n} / \partial \tau$} & \multicolumn{2}{c}{ Results for $\partial \phi_{1}^{n} / \partial \nu$} \\
\hline$n$ & Error & EOC & Error & EOC & Error & EOC \\
\hline 16 & $5.8841 \mathrm{e}-02$ & - & $7.029 \mathrm{e}-02$ & - & $9.647 \mathrm{e}-01$ & - \\
32 & $4.455 \mathrm{e}-03$ & 3.72 & $7.084 \mathrm{e}-03$ & 3.31 & $6.920 \mathrm{e}-02$ & 3.80 \\
64 & $2.517 \mathrm{e}-05$ & 7.47 & $5.694 \mathrm{e}-05$ & 6.96 & $4.186 \mathrm{e}-04$ & 7.37 \\
128 & $9.063 \mathrm{e}-10$ & 14.76 & $3.011 \mathrm{e}-09$ & 14.21 & $1.644 \mathrm{e}-08$ & 14.63 \\
256 & $1.847 \mathrm{e}-15$ & 18.90 & $2.762 \mathrm{e}-13$ & 13.41 & $3.618 \mathrm{e}-13$ & 15.47 \\
512 & $2.715 \mathrm{e}-15$ & -0.56 & $8.407 \mathrm{e}-13$ & -1.61 & $1.164 \mathrm{e}-12$ & -1.69 \\
\hline
\end{tabular}

show the results for the case of interface I1 in this form, which are presented in Table 1 . The results clearly reflect those shown in Figure 3. The estimated order of convergence (EOC) is computed via $\log _{2}(\operatorname{Error}(n) / \operatorname{Error}(n / 2))$, where we are referring to the discrete $l_{2}$ relative error as a function of $n$. The super-algebraic convergence is clear from the increasing EOC, which eventually reaches a value of around 17 in all cases before rounding errors begin to destroy the convergence rate when $n=256$.

Tables 2 and 3 show the relative error and EOC results for interfaces I2 and I3, respectively. Again, super-algebraic convergence is apparent until $n=256$, reaching peaks of almost order 19 in both cases. Once again, rounding errors begin to destroy the convergence rate when $n=256$. Both higher convergence rates and accuracy levels are observed in the computations of $\phi^{n}$ as compared with its directional derivatives. However, since the derivatives are computed from $\phi^{n}$, then this is probably to be expected; these trends are also consistent with the observations made in [19]. 
6. Conclusion. The transmission problem for the Laplace equation on a periodic half-space has been considered. The study was motivated by its application to the modelling of electrified oil films used in the development of novel switchable liquid optical devices (diffraction gratings). A boundary integral formulation which reduces the problem to the study of the interface alone was derived and solved in a highly efficient manner using the Nyström method. The quadrature rules were chosen with reference to supporting results in numerical analysis, and were predicted to converge super-algebraically. Numerical experiments demonstrated this convergence rate in practice for a choice of parameters appropriate to our goal application, and for a range of interface geometries.

Acknowledgments. The author thanks Prof. Carl Brown, Dr. Reuben O'Dea and Dr. B. Tomas Johansson for stimulating discussions. I am also indebted to the anonymous reviewers for their careful reading, suggestions and corrections.

\section{REFERENCES}

1. K.E. Atkinson, The numerical solution of integral equations of the second kind, Cambridge University Press, Cambridge, UK, 1997.

2. H.A.M Ben Hamdin, Boundary element and transfer operator methods for multi-component wave systems, Ph.D. thesis, University of Nottingham, UK, 2012.

3. H.A.M Ben Hamdin and G. Tanner, Multi-component BEM for the Helmholtz equation-A normal derivative method, Shock Vib. 19 (2012), 957-967.

4. C.V. Brown, G. McHale and N.J. Mottram, Analysis of a static undulation on the surface of a thin dielectric layer formed by dielectrophoresis forces, J. Appl. Phys. 110 (2011), 024107.

5. C.V. Brown, G.G. Wells, M.I. Newton and G. McHale, Voltage-programmable liquid optical interface, Nature Phot. 3 (2009), 403-405.

6. D.J. Chappell, G. Tanner and S. Giani, Boundary element dynamical energy analysis: A versatile method for solving two or three dimensional wave problems in the high frequency limit, J. Comp. Phys. 130 (2012), 6181-6191.

7. M. Costabel and E. Stephan, A direct boundary integral method for transmission problems, J. Math. Anal. Appl. 106 (1985), 367-413.

8. S.C. Creagh, H.A.M Ben Hamdin and G. Tanner, In-out decomposition of boundary integral equations, J. Phys. 46 (2013), 435203.

9. R.S. Falk and S.W. Walker, A mixed finite element method for EWOD that directly computes the position of the moving interface, SIAM J. Numer. Anal. 51 (2013), 1016-1040. 
10. G.C. Hsiao and L. $\mathrm{Xu}, A$ system of boundary integral equations for the transmission problem in acoustics, Appl. Num. Math. 61 (2011), 1017-1029.

11. T.B. Jones, M. Gunji, M. Washizu and M.J. Feldman, Dielectrophoretic liquid actuation and nanodroplet formation, J. Appl. Phys. 89 (2001), 1441.

12. R.E. Kleinman and P.A. Martin, On single integral equations for the transmission problem of acoustics, SIAM J. Appl. Math. 48 (1988), 307-325.

13. R. Kress, Linear integral equations, Second Edition, Springer, New York, NY, 1999.

14. R. Kress and I.H. Sloan, On the solution of a logarithmic integral equation of the first kind for the Helmholtz equation, Numer. Math. 66 (1993), 199-214.

15. C.M. Linton, Rapidly convergent representations for Green'functions for Laplace' equation, Proc. Roy. Soc. Lond. 455 (1999), 1767-1797.

16. H.A. Pohl, Some effects of non-uniform fileds on dielectrics, J. Appl. Phys. 29 (1958), 1182-1188.

17. Dielectrophoresis the behavior of neutral matter in nonuniform electric fields, Cambridge University Press, Cambridge, UK, 1978.

18. M.D. Preston, P.G. Chamberlain and S.N. Chandler-Wilde, An integral equation method for a boundary value problem arising in unsteady water wave problems, J. Integral Equat. Appl. 20 (2008), 113-143.

19. _ A Nyström method for a boundary value problem arising in unsteady water wave problems, IMA J. Numer. Anal. 31 (2011), 1123-1153.

20. M.-L. Rapun and F.J. Sayas, Mixed boundary integral methods for Helmholtz transmission problems, J. Comp. Appl. Math. 214 (2008), 238-258.

21. D. Volkov, D.T. Papageorgiou and P.G. Petropoulos, Accurate and efficient boundary integral methods for electrified liquid bridge problems, SIAM J. Sci. Comp. 26 (2005), 2102-2132.

22. S.W. Walker and B. Shapiro, Modeling the fluid dynamics of electro-wetting on dielectric (EWOD), J. Microelectromech. Syst. 15 (2006), 986-1000.

23. L.Y. Yeo and H.-C. Chang, Electrowetting films on parallel line electrodes, Phys. Rev. 73 (2006), 011605.

School of Science and Technology, Nottingham Trent University, Clifton Campus, Clifton Lane, Nottingham, UK NG11 8NS

Email address: david.chappell@ntu.ac.uk 